\title{
Analysis of a Clinically Failed, Mechanically Intact, Hemi-Toe Implant
}

\author{
Swetha Varadharajan ${ }^{1}$, Richard Laughlin'2, and Tarun Goswami ${ }^{1,2,3}$ \\ ${ }^{1}$ Department of Biomedical, Industrial and Human Factors Engineering, \\ Wright State University, Dayton, OH 45435, USA \\ ${ }^{2}$ Orthopaedic Surgery, Sports Medicine and Rehabilitation, \\ Wright State University, Dayton, OH 45435, USA \\ ${ }^{3}$ Institute of Materials Science and Welding, Graz University of Technology, \\ Kopernikusgasse 24/I, 8010 Graz, Austria \\ tarun.goswami@wright.edu
}

\begin{abstract}
In this case study a clinically failed, mechanically intact, hemi-toe device was investigated. The clinical indication of the failure constitutes radiolucent line indicating loosening of the implant and possible interactions among the boneimplant initiated by osteolysis may become a factor, producing pain, inflammatory reactions, deformity and discomfort. The patient file was not available to determine these parameters. The device was titanium coated, Cobalt Chromium alloy used for making hemi-toe. Degeneration of hemi implant was due to spalling of the coating from the surface, causing loosening of stem from bone. However, pre-removal X-ray films were not available to confirm. Mechanism by which the clinical failure occurred likely due to lack of bony adhesion or other clinical reasons. Surface topographical features were documented using optical microscope. A method to classify various area of the articulating surface was proposed in this study that shows the anterior contacts were more damaging than the other areas where deeper scratches, pits and some debris were present. There is a need to converge mechanical and clinical failures together for these types of medical devices.
\end{abstract}

Keywords: Hemi-Toe, Hallux toe, Fracture, metophalengeal joint.

(C) Copyright 2016 Authors - This is an Open Access article published under the Creative Commons Attribution License terms (http://creativecommons.org/licenses/by/3.0). Unrestricted use, distribution, and reproduction in any medium are permitted, provided the original work is properly cited.

\section{Introduction}

The hallux or great toe consists of metatarsal, and phalange forming a joint. The metaphalengeal joint is covered with articular cartilage. When cartilage wears out, or degenerated, bone-to-bone interaction occurs which results in decrease in movement of toe (1-2). As a result of this process, the person may experience pain in toe and surrounding areas, difficulty in rotation and other movement of toe, numbness, etc. A computed tomography image was imported in the Mimics (Materialise Inc, Leuven) and after thresholding and filtration, a 3D image of the big-toe joint was produced below. The joint developed in Fig. 1 clearly shows the complexity of the anatomical features and joint gap. It may be noted that this example is not a candidate for toe replacement devices.

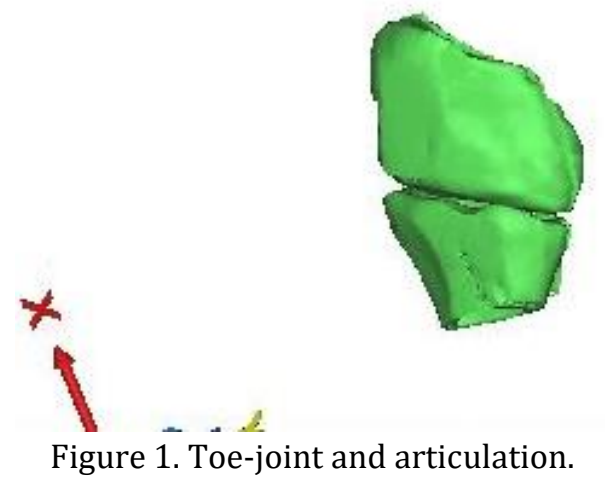

A toe implant replacement surgery is performed either by press-fit method for bony ingrowth to take place on the stem and the joint replacement restores movement and rotation of the toe joint (1-2). It can also be cemented. The present case was a press-fit device. 


\section{Materials and Methods}

The submitted device is shown in Figure 2. The stem is attached to the chord, whereas the convex surface articulates with a liner inserted on the metatarsal of the joint. The liner is made with ultra-high molecular weight polyethylene, and not submitted with the device. The implant is designed to support movement of the bone. The material used for this implant is cobalt chromium alloy. The use of coating is to improve the growth of tissues around the implant.

The dimensions of the hemi toe has been measured marked in Figure 2 (a), (b) and (c).
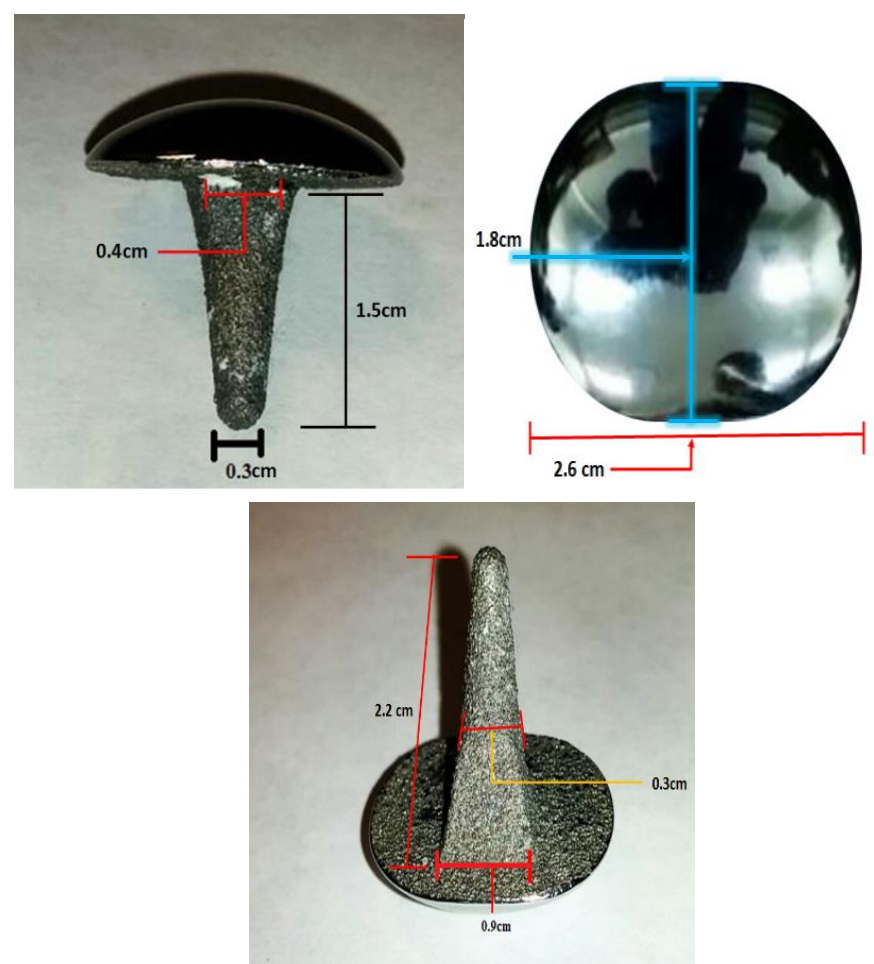

Figure 2. Device details from different angles, a) hemi-toe; b) posterior View of hemi-toe; c) bottom view of hemi-toe.

\section{1. Non-destructive testing}

The medical device was viewed under optical microscope at various magnifications. Following results were obtained during the process.

\section{1. 1. Visual observation}

Scratches were visible on the shiny articulating surface of hemi-cap. There are also visible wear, spalling off of coating from the stem of the implant, Fig. 3 . Visible sediments (white colored) on the stem and edges between stem and hemi-cap were notable. Since the stem was only presented for this investigation and liner was not available, the results and recommendations made in this paper constitute only for the metal stem.

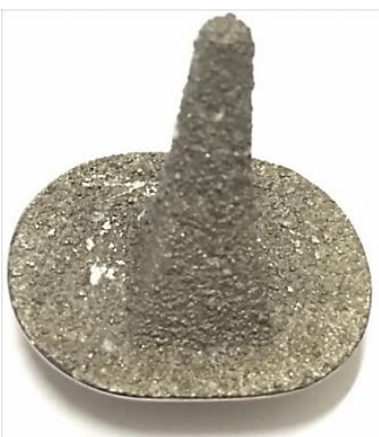

Figure 3. Wear and spalling of coating materials from surface.

\section{1. 2. Optical Microscopy}

The convex articulating surface of the hemi-toe implant was divided into 9 segments. These regions are marked accordingly, Figure 4.

- $\quad$ Anterior (A1, A2 an A3);

- $\quad$ Middle (M1, M2 and M3) and

- $\quad$ Posterior (P1, P2 and P3)

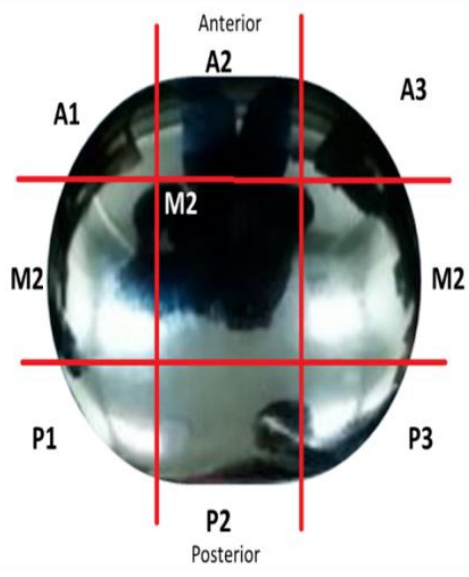

Figure 4. Nine segments marked on the articulating surface to assess damage.

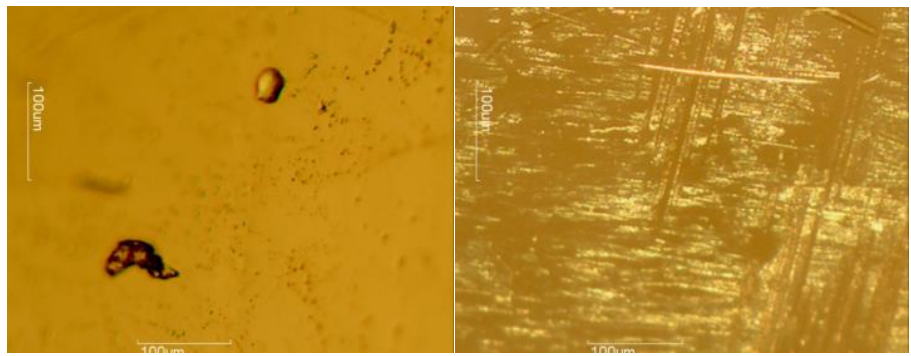

Figure 5. Optical microscope images at 40x shows pits and scratches in the middle regions. 


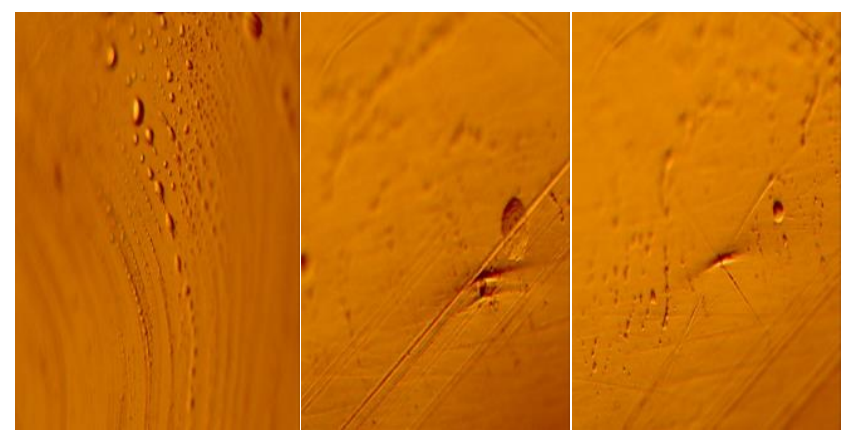

Figure 6. Optical microscope images on 40x magnification showing pits and scratches on the anterior and posterior regions where the hemi engages with liner A1, A2 and A3 regions.

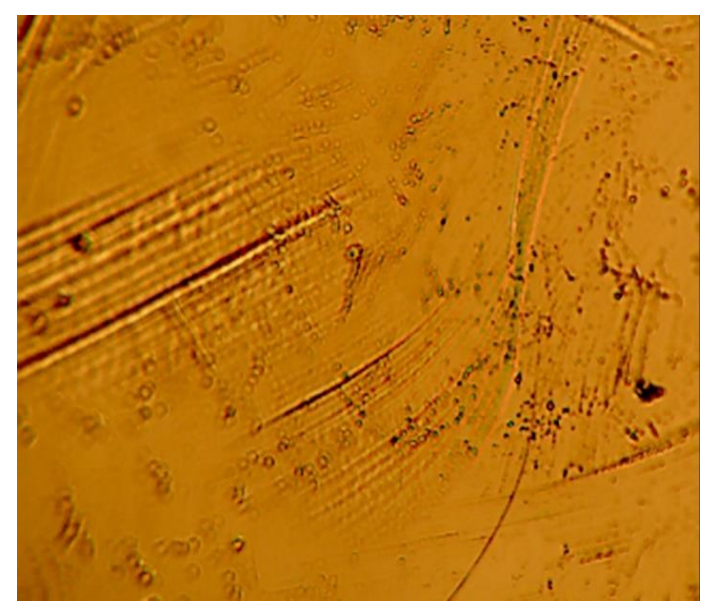

Figure 7. Optical microscope images at 40x showing pits, scratches on $\mathrm{A} 3$ region.

The coating on the surface of the stem was investigated. It appears that spherical beads were part of the coating system applied to the stem. The optical examination did not reveal biological matter in between the coating beads. Our experience has shown that biological matter builds in such areas, between the channels created by the beads.

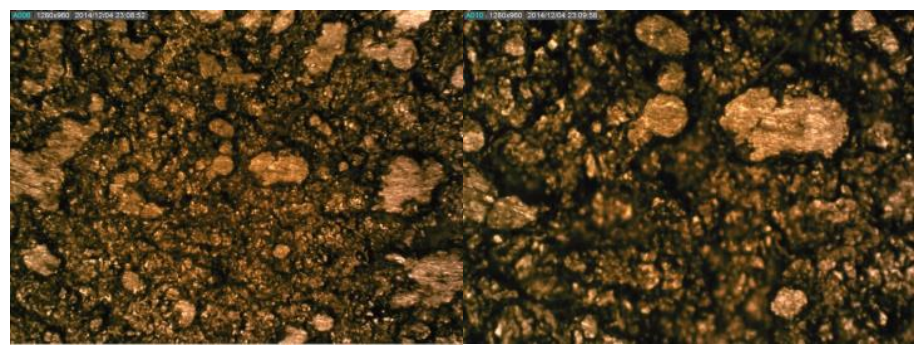

Figure 8. Spherical pattern of the coat (x15 and x18).

\section{Discussion}

The microscopy revealed that the anterior and middle section (Fig. 5-7) of the hemi experienced more scratches, wear and some debris deposition resulting from polymer debris and adhering to the surface may have occurred (3). Since the coating spalled (Fig. 3, 8) it may be possible that the device may have become loose. This loosening may have compromised the bony ingrowth and fixation of the device in the intramedullary canal of the bone. Since patient details are not available, it will not be possible to comment the time that this device was successful in vivo. The device maintained mechanical integrity though failed clinically because such fixations cause pain, deformity, infection and other potential problems (4).

The metallic implants usually articulate with the polymer bearing, it is possible that the bearing may have failed, another scenario, however, speculative. Bearing was not available for inspection in this study. However, it is also imperative that the degree of damage on the metal articulating surface may be indicative of higher damage on the bearing. This case shows that the metal surface was not severely damaged to cause the failure of the bearing.

This case provides a unique prospective to what constitutes a successful implantation and component integrity. Engineering prospective; though successful design, manufacturing and likelihood of durability, however, compromised in vivo, clinically. In the literature examples of toe replacement devices are not discussed, this study and others (4-5) provide opportunities for both communities to define this problem and converge engineering and clinical failures together.

\section{Conclusion}

The hemi-toe device was intact mechanically. The articulating surface did not reveal significant amount of damage either by wear, scratch, corrosion, and cracking. This study reveals that the damage was localized in the anterior contact points leading to the middle of the hemi surface.

It is possible that the liner, made of ultra-high molecular weight polyethylene may have failed due to manufacturing reasons or suffered impingement at the anterior area. However, in the absence of liner, it is speculated that, liner did not suffer extensive damage and removal.

It is recommended that the two communities, engineering and clinical, develop new standards to fully 
define what constitutes a failure more fully. This case is an example of successful failure, in that the implant component maintained the structural integrity, yet failed clinically.

\section{References}

[1] P. J. Kim, D. Hatch, L. A. Didomenico, M. S. Lee, B. Kaczander, G. Count and M. Kravette, "A multicenter retrospective review of outcomes for arthrodesis, hemimetallic joint implant, and resectional arthroplasty in the surgical treatment of end-stage hallux rigidus," Journal of Foot and Ankle Surgery, vol. 51, no. 1, pp. 50$56,2012$.

[2] Nixon, M. and G. Taylor, "Early failure of the Moje implant when used to treat hallux rigidus: the need for regular surveillance," The Foot, vol. 18, no. 1, pp. 1-6, 2008.

[3] T. Joyce, "Examination of failed ex vivo metal-onmetal metatarsophalangeal prosthesis and comparison with theoretically determined lubrication regimes," Wear, vol. 263, no. 7-12, pp. 1050-1054, 2007.

[4] N. Thapa and T. Goswami, "A failure study of a locking compression plate implant," Case Studies in Engineering Failure Analysis, vol. 3, pp. 68-72, 2015.

[5] S. Karmacharya and T. Goswami, "Analysis of a clinically failed, mechanically intact, reconstructive compression plate," Case Studies in Engineering Failure Analysis, 2015. 